\title{
Relationship between Clinical Outcomes of Patients Diagnosed with Pulmonary Thromboembolism in the Emergency Department and their PESI, Shock Index, Modified Shock Index and Age Shock Index Scores
}

\author{
ozkan erarslan ${ }^{1}$, Hayriye Gonullu ${ }^{1}$, serhat akay ${ }^{1}$, tayfun ozturk ${ }^{2}$, and mustafa silcan ${ }^{3}$ \\ ${ }^{1}$ Affiliation not available \\ ${ }^{2}$ İzmir Bozyaka Eğitim ve Araştırma Hastanesi \\ ${ }^{3}$ Kayseri City Education and Research Hospital
}

February 26, 2021

\begin{abstract}
Aim: To investigate the diagnostic value of pulmonary embolism severity index (PESI), Simplified PESI (sPESI), Shock Index (SI), Modified SI (MSI), and Age SI (ASI) scores in predicting 30-day mortality in patients diagnosed with pulmonary thromboembolism (PTE) in the emergency department. Materials and Methods: The retrospective study included 257 patients that presented to the emergency department and underwent contrast-enhanced computed tomography pulmonary angiogram (CTPA) due to the suspicion of PTE and were interpreted as PTE by an experienced radiologist between January 1, 2015 and September 20, 2018.The PESI, sPESI, SI, MSI, and ASI scores were calculated for each patient. Results: On univariate logistic regression analysis, 30-day mortality was found to be significantly associated with age, mode of presentation, SBP, DBP, MAP, heart rate, respiratory rate, O2 saturation, temperature, D-dimer, troponin I, high-sensitivity cardiac troponin (hs-cTn), lactate, and SI, MSI, ASI, PESI, and sPESI scores $(\mathrm{p}<0.05)$. PESI had the highest AUC value for the prediction of 30-day mortality among all scoring systems. Conclusion: PESI had the highest diagnostic value in predicting 30-day mortality in the patients diagnosed with PTE in the emergency department, followed by ASI. Our findings regarding ASI may shed light on future studies evaluating critical patient groups."
\end{abstract}

\section{Hosted file}

Manuscript.pdf available at https://authorea.com/users/398292/articles/510946-relationshipbetween-clinical-outcomes-of-patients-diagnosed-with-pulmonary-thromboembolism-in-theemergency-department-and-their-pesi-shock-index-modified-shock-index-and-age-shockindex-scores 